\title{
Factores de riesgo asociados a bacteriemia en lactantes. Servicio de Clínicas Pediátricas. 2019.
}

\section{Risk factors associated with bacteremia in infants. Pediatric Clinical Ward. 2019.}

\author{
Alitza Tamayo Cordoví. ${ }^{1}$ \\ Tamara Coto Batista. ${ }^{2}$ \\ Yelenis Elías Montes. $^{3}$ \\ Yusleidy Yanet Sánchez Pérez. ${ }^{4}$ \\ Annia María Linares Rodríguez. ${ }^{5}$
}

(1) Hospital Provincial Pediátrico Docente "General Milanés”. Bayamo. Granma, Cuba. email: alitc@infomed.sld.cu. ORCID: https://orcid.org/0000-0001-6760-9652

(2) Policlínico Docente Bayamo Oeste. Bayamo. Granma, Cuba, email: tcotob@infomed.sld.cu. ORCID: https://orcid.org/0000-0001-9891-8298

(3) Hospital Provincial Pediátrico Docente "General Milanés". Bayamo. Granma, Cuba. ORCID: https://orcid.org/0000-0002-5276-4956

(4) Hospital Provincial Pediátrico Docente "General Milanés". Bayamo. Granma, Cuba. ORCID: https://orcid.org/0000-0002-3741-7374

(5) Policlínico Docente 13 de Marzo. Bayamo. Granma, Cuba. email: anniamaria68@ gmail.com

Contacto: alitc@infomed.sld.cu

Recibido: 15-06-2020

Aprobado: 23-06-2020

\section{Resumen}

Introducción: la bacteriemia no tiene nada que ver con algo tóxico. Se considera una etapa temprana de la sepsis. Objetivo: identificar algunos factores de riesgo que se asociaron a bacteriemia en lactantes ingresados en el servicio de clínicas pediátricas del Hospital Provincial Pediátrico Docente "General Milanés" en el período comprendido del $1^{\text {ro }}$ enero al 31 diciembre 2019, en Granma. Método: se realizó un estudio observacional analítico de caso y control que investigó la asociación entre la bacteriemia y factores de riesgo relacionados con la edad, sexo, desnutrición, bajo peso al nacer, prematuridad, no lactancia materna exclusiva, uso previo de antimicrobianos y hospitalización reciente. Se seleccionaron 48 casos y 144 controles. Se utilizaron métodos teóricos y empíricos y se calculó el Odd Ratio. Resultados: en el análisis univariado la edad $(\mathrm{OR}=2,51, \mathrm{IC}=1,28-4,91 ; \mathrm{p}=0,01)$, la desnutrición $(\mathrm{OR}=30,88, \quad \mathrm{p}=0,00 ; \quad \mathrm{IC}=8,28-115,17)$, la prematuridad $(\mathrm{OR}=71,51, \mathrm{p}=0,00 ; \mathrm{IC}=13,55-77,35)$, el bajo peso al nacer $(\mathrm{OR}=6,90, \mathrm{p}=0,01 \mathrm{IC}=3,27$ $14,58)$, la no lactancia materna exclusiva $(\mathrm{OR}=6,90$, $\mathrm{IC}=3,15-15,12 ; \quad \mathrm{p}=0,00)$, el uso previo de antimicrobianos $\quad(\mathrm{OR}=19,67, \quad \mathrm{IC}=7,03-55,06$, $\mathrm{p}=0,00)$ y la hospitalización reciente $(\mathrm{OR}=16,75$, $\mathrm{IC}=6,45-43,40 ; \mathrm{p}=0,00)$ contribuyeron al riesgo de bacteriemia en lactantes. El sexo no tuvo relación estadísticamente significativa $(\mathrm{OR}=1,55, \mathrm{IC}=0,81$ 2,98; $\mathrm{p}=0,24)$. Conclusiones: La hospitalización reciente y la desnutrición fueron los de mayor riesgo. Se concluye que con los valores del modelo de la regresión logística binaria, se podrá estimar el riesgo de padecer esta enfermedad.

Palabras clave: factores de riesgo, bacteriemia, lactantes

\begin{abstract}
Introduction: bacteremia has nothing to do with something toxic. It is considered a sepsis's premature stage. Objective: to identify some risk factors related with the bacteremia in infants that were admitted to the pediatric clinical ward of the Provincial Pediatric Teaching Hospital "General Milanés" in the period from January to December 2019, in Granma. Method: an observational analytical case and control study was carried out that investigated the association between bacteremia and risk factors related to the age, sex, malnutrition, under birth weight, prematurity, not exclusive breast-feeding, antimicrobial therapy use and recent hospitalization. Forty-eight cases and one hundred forty four controls were selected. Theoretical and empirical methods
\end{abstract}


were used and the Odd Ratio was calculated. Results: age $(\mathrm{OR}=2,51, \mathrm{IC}=1,28-4,91 ; \mathrm{p}=0,01)$, malnutrition $(\mathrm{OR}=30,88, \mathrm{p}=0,00 ; \mathrm{IC}=8,28-115,17)$, under birth weight $(\mathrm{OR}=6,90, \mathrm{p}=0,01 \mathrm{IC}=3,27$ $14,58)$, prematurity $(\mathrm{OR}=71,51, \mathrm{p}=0,00$; $\mathrm{IC}=13,55$ $77,35)$, not exclusive breast-feeding ( $\mathrm{OR}=6,90$, $\mathrm{IC}=3,15-15,12 ; \mathrm{p}=0,00)$, antimicrobial therapy use $(\mathrm{OR}=19,67, \mathrm{IC}=7,03-55,06, \mathrm{p}=0,00)$ and recent hospitalization $(\mathrm{OR}=16,75, \mathrm{IC}=6,45-43,40 ; \mathrm{p}=0,00)$ contributed to the risk of bacteremia in infants. The sex had no significant statistical relationship. Conclusions: the recent hospitalization and malnutrition were the factors of greatest risk. It is concluded that with the values of the binary logistic regression model, the risk of suffering from this disease can be estimated.

Keywords: risk factors, bacteremia, infants

\section{Introducción}

Varios estudios de investigación han recalcado la importante contribución de las infecciones bacterianas adquiridas en la comunidad en la morbilidad y mortalidad de los pacientes, si bien la mayoría de estos estudios se han llevado a cabo en la población adulta, los pocos estudios realizados en niños, confirman el rol de las bacteriemias en la gravedad, sobre todo en menores de 5 años de edad. (Araya et al., 2018)

La bacteriemia se define como la presencia de bacterias en sangre. (Blázquez Abellán, 2016; Reisde-Santana, Lima, Fraga, \& Gonçalves, 2016; Torres Hernández, García Gómez, Pradere Pensado, Rives, \& Fernández Castillo, 2019; "Willis-Knishton health system. Bacteriemia en niños," 2020). La invasión del torrente circulatorio por microorganismos puede producirse desde un foco infeccioso extravascular mediante el sistema linfático y directamente desde focos intravasculares (endocarditis, infecciones de catéteres intravasculares). (Blázquez Abellán, 2016)

Las bacteriemias pueden presentar 3 patrones clínicos:

- Bacteriemia transitoria: aquella que tiene lugar tras la manipulación de tejidos infectados (absceso, forúnculos, cirugía) o la instrumentación de superficies mucosas (endoscopias, cistoscopias, etc.) y dura de minutos a horas.

- Bacteriemia intermitente o "de brecha": ocurre durante un tratamiento antimicrobiano apropiado y en la que unos hemocultivos previos son negativos. Es típica de infecciones cerradas como los abscesos intraabdominales.

- Bacteriemia continua: cuando los hemocultivos se mantienen positivos después de 48-96h horas de tratamiento adecuado, es característica de infecciones endovasculares como las endocarditis y la tromboflebitis supurada. (Blázquez Abellán, 2016)

Se define como bacteriemia oculta (BO) a la presencia de una bacteria en sangre en un niño febril previamente sano, que luce bien, y cuyas condiciones clínicas permiten tratarlo de forma ambulatoria en la mayoría de los pacientes. La frecuencia de esta enfermedad se encuentra en el $4 \%$ de los niños con buen aspecto que tienen de tres a 36 meses de edad y presentan fiebre de $38^{\circ} \mathrm{C}$. (Yaguache Hernández, 2015)

En general la bacteriemia oculta no presenta complicaciones graves, su evolución es transitoria y la resolución, en la mayoría de los casos, es espontánea sin usar antibióticos, pero el 11,7\% puede dar lugar a infecciones graves. (Covián, Limón, Valenzuela, Ortiz, \& Reyna, 2014)

La incidencia de infección del torrente sanguíneo en Europa es más de 1 millón de casos y 157 mil muertes por año, en Norte América más de 650 mil episodios por año y las muertes anuales supera los 70 mil casos. (Laurente Sáenz, 2020)

Se estima que en Nueva Zelanda y Canadá, la incidencia en niños con bacteriemia por Estafilococcus aureus adquiridas en la comunidad (BSAC) es 16,9 y 17,9 casos por cada 100000 personas, respectivamente; mientras en un hospital pediátrico de Argentina 1,08 casos por cada 1000 ingresos hospitalarios. (Araya et al., 2018)

Desde el punto de vista de la mortalidad infantil, la bacteriemia no constituye un problema de salud en Cuba, puesto que las tasas de mortalidad que con ellas se asocian en menores de 5 años y otros grupos de edad son comparables a las de los países desarrollados. Se ha logrado reducir la mortalidad por bacteriemia mediante diferentes medidas tales como: incremento de la lactancia materna, uso racional de antibióticos, capacitación de los recursos humanos y manejo correcto de las mismas. (PérezRodríguez, 2013) 
Cuando se analizan los factores de riesgos que contemplan la bacteriemia en la población pediátrica, que inciden en la gravedad y la mortalidad del paciente al momento del ingreso hospitalario, será de gran utilidad para lograr Estrategias Preventivas de Salud Pública, las que tendrán un impacto positivo en la sobrevida de estos niños.

Se define como objetivo general, identificar los factores de riesgo asociados al desarrollo de bacteriemia en lactantes.

Se trataron como objetivos específicos: determinar la posible asociación de la edad, sexo, desnutrición, bajo peso al nacer, prematuridad, no lactancia materna exclusiva, uso previo de antimicrobianos y hospitalización reciente con la aparición de bacteriemia en lactantes.

\section{Método}

Se realizó un estudio observacional analítico tipo caso y control con el objetivo de identificar la influencia de algunos factores de riesgo asociados al desarrollo de bacteriemia en lactantes ingresados en el servicio de clínicas pediátricas del Hospital Provincial Pediátrico Docente "General Milanés" en Bayamo, Granma, en el período comprendido del $1^{\text {ro }}$ enero al 31 diciembre 2019.

Se incluyeron aquellos lactantes con manifestaciones clínicas y humorales de infección que tuvieron aislamiento en hemocultivo, ingresados en el servicio de clínicas pediátricas del hospital donde se llevó a cabo el estudio y voluntariedad para participar por parte de los tutores mediante el consentimiento informado.

Se excluyeron de la investigación los lactantes que tuvieron aislamiento en hemocultivo pero no presentaron síntomas de infección y aquellos pacientes con enfermedades asociadas como anemia hemolítica, fibrosis quística, tumores, malformaciones congénitas e inmunodepresión.

La selección de los casos estuvo formada por los pacientes menores de 1 año que ingresaron en el servicio de clínicas pediátricas que tuvieron aislamiento en hemocultivo y que presentaron síntomas clínicos y humorales de infección y identificar los factores de riesgo asociados al desarrollo de bacteriemia en lactantes.

Período de estudio. La selección de los casos se llevó a cabo durante el año de estudio, quedando conformada por 48 pacientes.

Se seleccionaron como controles aquellos pacientes que ingresaron en el mismo servicio que los casos con otro diagnóstico, que tenían la misma edad y que ingresaron consecutivamente.

Se seleccionaron 3 controles por cada caso (1:3) quedando constituido por un total de 144 controles.

Los datos necesarios para el estudio se recogieron de las historias clínicas hospitalarias y de entrevistas semi-estructuradas a los tutores, con la utilización de un formulario mediante el cual se obtuvo el comportamiento de las diferentes variables objeto de estudio: edad, sexo, desnutrición, bajo peso al nacer, prematuridad, lactancia materna exclusiva, uso previo de antimicrobianos; así como la hospitalización reciente.

Se tuvieron en cuenta los principios éticos de la investigación biomédica. Además de las regulaciones estatales del Ministerio de Salud Pública (MINSAP), vigentes en la República de Cuba. Para la evaluación de los factores de riesgo, se utilizó el programa EPIDAT versión 3.1. Se realizó unanálisis univariado, se determinó el Odds ratio (OR), considerándose como factor de riesgo, cuando el OR poblacional, fuese mayor o igual de 1.01. Se tuvo en cuenta el intervalo de confianza igual al 95 $\%$, con un nivel de significación de $0,05(\mathrm{p}<0,05)$. Los resultados fueron expresados en tablas para su mejor comprensión.

\section{Resultados}

En el análisis univariado, relacionado con la edad se encontró que el grupo de 1-5 meses y 29 días duplicó el riesgo de enfermar por bacteriemia $(\mathrm{OR}=2,51, \mathrm{IC}=$ $1,28$ - 4,91; $\mathrm{p}=0,01)$ y el sexo masculino incrementó el riesgo $(\mathrm{OR}=1,55, \mathrm{IC}=0,81-2,98 ; \mathrm{p}=0,24)$; $\sin$ embargo, este último no tuvo significación estadística. (Tabla 1) 


\begin{tabular}{|c|c|c|c|c|c|c|c|}
\hline \multirow{2}{*}{\multicolumn{2}{|c|}{ Variables }} & \multirow{3}{*}{$\begin{array}{l}\text { Casos } \\
\mathrm{N}=48 \\
31 \\
\end{array}$} & \multirow{3}{*}{$\begin{array}{l}\text { Controles } \\
N=144 \\
60\end{array}$} & \multirow{4}{*}{$\begin{array}{l}\text { OR } \\
2,51\end{array}$} & \multicolumn{2}{|c|}{$\begin{array}{l}\text { Intervalo de confianza } \\
95 \%\end{array}$} & \multirow{4}{*}{$\begin{array}{l}P \\
0.01\end{array}$} \\
\hline & & & & & Mín. & Máx. & \\
\hline \multirow{2}{*}{ Edad } & $1-5 \mathrm{~m}$ y 29 días & & & & 128 & 491 & \\
\hline & 6-11m y 29 días & 17 & 84 & & 1,28 & 4,91 & \\
\hline \multirow[t]{2}{*}{ Sexo } & Masculino & 26 & 64 & \multirow{2}{*}{1,55} & \multirow{2}{*}{0,81} & \multirow{2}{*}{2,98} & \multirow{2}{*}{0,24} \\
\hline & Femenino & 22 & 80 & & & & \\
\hline
\end{tabular}

Tabla 1. Factores de riesgo de bacteriemia según edad y sexo. Hospital Provincial Pediátrico Docente "General Milanés”. 2019. Fuente: Historia clínica

En la tabla 2, se representa el análisis referente a la desnutrición, peso al nacer y prematuridad mostrando las tres asociaciones estadísticas. La desnutrición elevó treinta veces el riesgo de enfermar por bacteriemia $(\mathrm{OR}=30,88, \mathrm{p}=0,00 ; \mathrm{IC}=8,28$ 115,17), continuándole la prematuridad, la cual elevó a casi setenta y dos veces la posibilidad de adquirir la enfermedad $(\mathrm{OR}=71,51, \mathrm{p}=0,00 ; \mathrm{IC}=13,55$ 77,35). Relacionado con el peso al nacer, el haber nacido bajo peso elevó 6 veces el riesgo $(\mathrm{OR}=6,90$, $\mathrm{p}=0,01 \mathrm{IC}=3,27-14,58)$.

\begin{tabular}{|c|c|c|c|c|c|c|c|}
\hline \multirow{2}{*}{\multicolumn{2}{|c|}{ Variables }} & \multirow{2}{*}{$\begin{array}{l}\text { Casos } \\
\mathrm{N}=48\end{array}$} & \multirow{2}{*}{$\begin{array}{l}\text { Controles } \\
\mathrm{N}=144\end{array}$} & \multirow[t]{2}{*}{ OR } & \multicolumn{2}{|c|}{$\begin{array}{l}\text { Intervalo de } \\
\text { confianza } 95 \%\end{array}$} & \multirow[t]{2}{*}{$\mathrm{P}$} \\
\hline & & & & & Mín. & Máx. & \\
\hline \multirow{2}{*}{ Desnutrición } & $\mathrm{Si}$ & 46 & 54 & \multirow{2}{*}{30,88} & \multirow{2}{*}{8,28} & \multirow{2}{*}{115,17} & \multirow{2}{*}{0,00} \\
\hline & No & 2 & 90 & & & & \\
\hline \multirow[b]{2}{*}{ Peso al nacer } & Bajo peso & 37 & 46 & \multirow{2}{*}{6,90} & \multirow{2}{*}{3,27} & \multirow{2}{*}{14,58} & \multirow{2}{*}{0,01} \\
\hline & Normopeso & 11 & 98 & & & & \\
\hline \multirow{2}{*}{ Prematuridad } & $\mathrm{Si}$ & 47 & 44 & \multirow[t]{2}{*}{71,51} & \multirow{2}{*}{13,55} & \multirow[t]{2}{*}{77,35} & \multirow{2}{*}{0,00} \\
\hline & No & 1 & 100 & & & & \\
\hline
\end{tabular}

Tabla 2. Factores de riesgo de bacteriemia según desnutrición, peso al nacer y prematuridad. Hospital Provincial Pediátrico Docente "General Milanés". 2019. Fuente: Historia clínica

Relacionado con la lactancia materna se identifica asociación significativa entre la no lactancia materna exclusiva y la bacteriemia, elevando a más de 6 veces el riesgo $(\mathrm{OR}=6,90, \mathrm{IC}=3,15-15,12 ; \mathrm{p}=0,00)$, el uso previo de antimicrobianos eleva a más de diecinueve veces la probabilidad $(\mathrm{OR}=19,67, \mathrm{IC}=7,03-55,06$, $\mathrm{p}=0,00)$ y la hospitalización reciente a más de dieciséis veces $(\mathrm{OR}=16,75, \mathrm{IC}=6,45-43,40 ; \mathrm{p}=0,00)$; quedando demostrado que todos se comportaron como factores de riesgo (tabla 3).

\begin{tabular}{|c|c|c|c|c|c|c|c|}
\hline \multirow{2}{*}{\multicolumn{2}{|c|}{ Variables }} & \multirow{2}{*}{$\begin{array}{l}\text { Casos } \\
\mathrm{N}=48\end{array}$} & \multirow{2}{*}{$\begin{array}{l}\text { Controles } \\
\mathrm{N}=144\end{array}$} & \multirow{2}{*}{ OR } & \multicolumn{2}{|c|}{$\begin{array}{l}\text { Intervalo de } \\
\text { confianza 95\% } \\
\end{array}$} & \multirow{2}{*}{$\mathrm{P}$} \\
\hline & & & & & Mín & Máx & \\
\hline \multirow{2}{*}{ Lactancia materna } & No Exclusiva & 39 & 54 & \multirow{2}{*}{6,90} & \multirow{2}{*}{3,15} & \multirow{2}{*}{15,12} & \multirow{2}{*}{0,00} \\
\hline & Exclusiva & 9 & 90 & & & & \\
\hline \multirow{2}{*}{$\begin{array}{l}\text { Uso previo de } \\
\text { antimicrobiano }\end{array}$} & $\mathrm{Si}$ & 44 & 48 & \multirow{2}{*}{19,67} & \multirow{2}{*}{7,03} & \multirow{2}{*}{55,06} & \multirow{2}{*}{0,00} \\
\hline & No & 4 & 96 & & & & \\
\hline \multirow{2}{*}{$\begin{array}{l}\text { Hospitalización } \\
\text { reciente }\end{array}$} & $\mathrm{Si}$ & 43 & 46 & \multirow{2}{*}{16,75} & \multirow{2}{*}{6,45} & \multirow{2}{*}{43,40} & \multirow{2}{*}{0,00} \\
\hline & No & 5 & 98 & & & & \\
\hline
\end{tabular}

Tabla 3. Factores de riesgo de bacteriemia según lactancia materna, uso de antimicrobianos y hospitalización reciente. Hospital Provincial Pediátrico Docente "General Milanés". 2019. Fuente: Historia clínica.

El análisis multivariado muestra que todos de los factores de riesgos estudiados incrementaron el riesgo de presentar bacteriemia. Los factores de mayor riesgo fueron la hospitalización reciente $(\mathrm{OR}=19,08, \mathrm{IC}=4,56-63,43 ; \mathrm{p}=0,03)$ y la desnutrición $(\mathrm{OR}=13,31, \mathrm{IC}=2,50-66,17 ; \mathrm{p}=0,01)$; estadísticamente significativas con el riesgo de enfermar por bacteriemia. (Tabla 4) 


\begin{tabular}{|l|l|l|l|l|}
\hline Factores de riesgo & P & OR & \multicolumn{2}{l|}{ IC. 95,0\% } \\
\cline { 4 - 5 } & & & Inferior & Superior \\
\hline Edad de 1-5m y 29días & 0,02 & 1,61 & 0,56 & 4,62 \\
\hline Sexo & 0,02 & 1,61 & 2,30 & 13,99 \\
\hline Desnutrición & 0,01 & 13,31 & 2,50 & 66,17 \\
\hline Bajo peso al nacer & 0,01 & 2,64 & 1,57 & 5,83 \\
\hline Prematuridad & 0,00 & 3,27 & 1,06 & 10,08 \\
\hline Lactancia Materna Exclusiva & 0,00 & 3,50 & 1,74 & 7,20 \\
\hline Uso previo de antimicrobianos & 0,00 & 1,05 & 0,25 & 5,24 \\
\hline Hospitalización Reciente & 0,03 & 19,08 & 4,56 & 63,43 \\
\hline
\end{tabular}

Tabla 4. Factores de riesgo de bacteriemia en lactantes. Hospital Provincial Pediátrico Docente "General Milanés". 2019. Análisis multivariado.

\section{Discusión}

En el presente estudio la población menor de 6 meses es la más afectada, lo cual se atribuye a que los lactantes tienen un riesgo más elevado de padecer infecciones que los niños mayores, ya que tienen un estado inmunológico deteriorado y menor respuesta a la agresión contra agentes externos, que los hace más susceptibles.

En un estudio realizado en el Hospital Nacional de Niños en Costa Rica el grupo de edad de 0 a 30 días es el que presentó mayor número de infecciones en un $40 \%$ y $66 \%$, en el año 2012 y 2013 respectivamente; comportándose como el grupo más vulnerable $^{(10)}$; coincidiendo la presente serie en que el menor de 6 meses tiene mayor riesgo de padecer de infecciones. Sin embargo, en otra investigación los niños de 1-4 años fue el grupo más representativo relacionado con las bacteriemias adquiridas en la comunidad. (Araya et al., 2018)

En los primeros 2-3 meses de vida, el sistema inmune es relativamente inmaduro. $\mathrm{La}$ respuesta quimiotáctica, opsonización, actividad macrofágica y de neutrófilos están disminuidas, haciendo al lactante más susceptible de padecer enfermedad bacteriana potencialmente grave (EBPG). Sumado a esto, la escasez de síntomas aún en casos de infección bacteriana severa, hace difícil su abordaje. (Peruffo et al., 2017)

En esta investigación el sexo más afectado fue el masculino y en estudios realizados por otros autores se presentan resultados similares. (Correa Gómez; Reis-de-Santana et al., 2016; Sánchez, Jiménez, \& de Mezerville, 2014)

En cuanto a la población pediátrica, en una tesis relacionada con Bacteriemias por Staphylococcus Aureus; predominó el sexo femenino con 11 pacientes (52 \%) (Blázquez Abellán, 2016); no mostrándose coincidencia entre ambos estudios.

La teoría propuesta más aceptada en relación a este fenómeno es que las hembras, al poseer dos cromosomas X tendrán mayor protección contra las infecciones ya que un gen localizado en el cromosoma $\mathrm{X}$ está relacionado con la función del timo y secreción de inmunoglobulinas. (Huete Gaitán, 2016)

Si se tiene en consideración que algunos de los niños nacidos con bajo peso no alcanzan en su primer año de vida un percentil de acuerdo con su peso/talla, se puede comprender que se sumaría otro factor predisponente para las bacteriemias, aparejado a la malnutrición y al déficit inmunológico que presentan en su mayoría.

El niño que tiene un peso bajo al nacer, donde solo por el hecho de nacer con un peso menor a 2500 gramos el riesgo de padecer infecciones es dos veces mayor que el de uno con un peso mayor. Puesto a mayor prematuridad y menor peso mayor será la inmadurez inmunológica; el paciente en este caso no habrá recibido las inmunoglobulinas maternas, estas atraviesan la placenta a partir de la 32 semana gestacional.

Los recién nacidos prematuros (nacidos entre la semana 22-36 de gestación) tiene 5 veces más riesgo de desarrollar una infección en relación con los nacidos en la semana 37 o mayor a esta. (Huete Gaitán, 2016)

Un estudio realizado en el año 2018, plantea que la prematuridad no se asoció con bacteriemia en la población pediátrica del estudio, lo que sugiere que el sistema inmunitario y los órganos en niños nacidos prematuramente maduran adecuadamente durante la infancia, por lo tanto, el nacimiento prematuro no 
confiere un factor de riesgo más adelante en la vida. (Pérez et al., 2016)

Las alteraciones inmunitarias están relacionadas con la edad gestacional; mientras mayor sea el grado de prematuridad, mayor es la inmadurez inmunológica $\mathrm{y}$, por ende, aumenta el riesgo de infección. La transferencia placentaria materna de $\mathrm{IgG}$ al feto comienza a las 32 semanas de gestación. El recién nacido depende por lo tanto de anticuerpos maternos pasivamente adquiridos, los cuales son transmitidos vía transplacentaria desde las 24 a las 26 Semanas de gestación. Los niños prematuros tienen significativamente menores niveles de anticuerpos IgG que los niños nacidos a término. (Huete Gaitán, 2016)

Por otro lado, más de la mitad de los casos tuvo un estado de desnutrición con diferencias estadísticamente significativas.

La desnutrición constituye la causa más frecuente de afectación del sistema inmune. Esto se explica porque durante los procesos infecciosos, se producen cambios metabólicos y se conoce que las deficiencias nutricionales individuales, tienen influencias marcadas, en el sistema inmune, pues la labilidad del patógeno para colonizar el hospedero, su mecanismo de adhesión y virulencia, juegan un papel importante; lo que puede facilitar el establecimiento de enfermedades, por producir cambios importantes en la estructura y función del hospedero. (PérezRodríguez, 2013)

El presente trabajo mostró que la ausencia de la lactancia materna exclusiva constituyó un factor de riesgo de adquirir bacteriemia.

No se encontraron estudios para comparar cómo influye la no lactancia materna exclusiva en la aparición de bacteriemia.

Esto puede atribuirse a que durante los primeros seis meses la leche materna se considera el alimento ideal; es la primera vacuna a la que se enfrenta el bebé cuando nace, con gran valor inmunológico que le transfiere la madre al niño y que favorece la prevención de enfermedades infecciosas.

De los otros factores de riesgo estudiados, el uso previo de antibiótico reveló ser altamente significativo.

En un estudio sobre bacteriemia por pseudomona aeruginosa en niños, el $85 \%$ habían recibido antibióticos en el último mes. (Rosaniva et al., 2018)
De igual manera, se encontró episodios de bacteriemia en adultos en el $11,5 \%$ de los casos, después de haber recibido tratamiento antimicrobiano reciente. (Torres Hernández et al., 2019)

La terapia antimicrobiana previa produce un incremento de la resistencia en los microorganismos, que se puede explicar por un desplazamiento a favor de especies bacterianas de mayor resistencia intrínseca o bien por un aumento de la resistencia adquirida en cepas de las mismas especies. En adición, la exposición previa a antibióticos aumenta el riesgo de subsiguiente fungemia (PérezRodríguez, 2013); lo que explica la asociación del uso previo de antibiótico y la bacteriemia.

Se debe enfatizar en la importancia de los programas de control de antimicrobianos y la vigilancia, para garantizar que los antimicrobianos se usen de manera empírica exclusivamente en pacientes con factores de riesgo. Además, el tratamiento deberá ajustarse cuando se pueda reducir el espectro antimicrobiano con base en el resultado de los cultivos. (Valderrama et al., 2016)

Las bacteriemias se asocian a un incremento de los días de hospitalización y a un aumento del costo derivado de la prolongación de la estancia hospitalaria y de las exploraciones complementarias asociadas. (Blázquez Abellán, 2016)

En un estudio presentado, la bacteriemia por Acinetobacter baumannii fue más frecuente en pacientes con mayor tiempo de internación en Unidad de cuidados intensivos constituyendo un factor de riesgo. (Ruvinsky et al., 2015)

La hospitalización implica un riesgo de adquirir bacteriemia debido a que en los niños, la susceptibilidad varía de acuerdo con el grupo de edad y el grado de madurez. (Pérez-Rodríguez, 2013)

\section{Conclusiones}

La mayoría de los factores investigados contribuyeron al riesgo de enfermar por bacteriemia, excepto el sexo. La hospitalización reciente y la desnutrición fueron los de mayor significación. Se concluye que con los valores del modelo de la regresión logística binaria, se podrá estimar el riesgo de padecer esta enfermedad.

\section{Referencias bibliográficas}


Araya, S., Troche, A., Amarilla, S., Sanabria, G., Zarate, C., Galeano, F., . . . Apodaca, S. (2018). Factores pronósticos de mortalidad en niños con bacteriemias adquiridas en la comunidad (BAC) hospitalizados en un centro de referencia del Paraguay. Pediatría (Asunción), 45(1), 17-24. Retrieved from http://scielo.iics.una.py/pdf/ped/v45n1/16839803-ped-45-01-17.pdf

Blázquez Abellán, A. (2016). Bacteriemias por staphylococcus aureus en el Hospital Clínico Universitario Virgen de la Arrixaca: estudio epidemiológico, clínico y molecular. Proyecto de investigación: Retrieved from: https://digitum.um.es/digitum/bitstream/1020 1/49423/1/Ana\%20B1\%c3\%a1zquez\%20Abe $11 \%$ c3\%a1n\%20Tesis\%20Doctoral.pdf

Correa Gómez, J. M. Factores de riesgo para bacteriemia asociada a Cateter en población Pediátrica de la fundación Hospital de la Misericordia. Retrieved from:

http://www.bdigital.unal.edu.co/50925/1/109 8649384.2015.pdf

Covián, M., Limón, A., Valenzuela, E., Ortiz, J., \& Reyna, J. (2014). Identificación de bacteremia oculta del lactante utilizando la prueba de Septifast: a propósito de un caso. Enfermedades Infecciosas y Microbiología, 34(2), 69. Retrieved from:

https://www.researchgate.net/profile/Federic o_Ortiz-

Ibarra/publication/283594013_Identification _of_occult_bacteriemia_in_infants_using_Se ptiFast_Case_report/links/5b7b68944585151 fd123d4ab/Identification-of-occultbacteriemia-in-infants-using-SeptiFast-Casereport.pdf

Huete Gaitán, J. F. (2016). Factores de riesgo asociados a Sepsis Neonatal en Recién Nacidos atendidos en el Hospital Primario Esteban Jaenz Serrano, Bonanza-RAANMinas, durante el periodo comprendido del 1 de Enero al 1 de Julio del año 2015. Universidad Nacional Autónoma de Nicaragua, Managua. Retrieved from http://repositorio.unan.edu.ni/3574/1/60438. pdf

Laurente Sáenz, M. (2020). Bacteriemia en los pacientes del Hospital Nacional Ramiro
Prialé Prialé de Huancayo 2017-2019. Retrieved from:

https://repositorio.uncp.edu.pe/bitstream/han dle/UNCP/5823/LAURENTE\%20SAENZ.p df? sequence $=1 \&$ is Allowed $=y$

Pérez-Rodríguez, Y. (2013). Factores de riesgo de bacteriemias en lactantes. Bayamo. BayamoGranma: Hospital Pediátrico Docente "General Luis Ángel Milanés Tamayo"; 2013. 31h. Bayamo-Granma: Hospital Pediátrico Docente "General Luis Ángel Milanés Tamayo"; 2013. 31h. .

Pérez, G., Martiren, S., Reijtman, V., Romero, R., Mastroianni, A., Casimir, L., \& Bologna, R. (2016). Bacteriemia por Staphylococcus aureus adquirido en la comunidad en niños: estudio de cohorte 2010-2014. Archivos argentinos de pediatría, 114(6), 0-0. Retrieved from: https://www.sap.org.ar/docs/publicaciones/ar chivosarg/2016/v114n6a06.pdf

Peruffo, M., Nainsztein, G., Maffia, S., Samaruga, C., Salvaneschi, V., \& Malvaso, R. (2017). Síndrome Febril sin foco en niños menores de meses. Ludovica pediátr, 20(1), 9-18.

Reis-de-Santana, T., Lima, A. A., Fraga, I., \& Gonçalves, J. (2016). Infecção de corrente sanguínea em um hospital terciário. Rev Soc Bras Clin Med, 14(1), 22-26. Retrieved from: http://www.sbcm.org.br/ojs3/index.php/rsbc $\mathrm{m} /$ article/view/188

Rosaniva, M., Mussini, M., Arias, A., Sormani, M., Mastroianni, A., \& García, M. (2018). Bacteriemia por Pseudomonas aeruginosa (PAE) en niños. Medicina Infantil, 25(4), 299-302. Retrieved from: www.medicinainfantil.org.ar/images/stories/ volumen/2018/xxv_4_299.pdf

Ruvinsky, S., Fiorilli, G., Pérez, M. G., Motto, E., Cambaceres, C., Mannino, L., . . . Bologna, R. (2015). Factores de riesgo para la adquisición y características microbiológicas de las bacteriemias por Acinetobacter baumannii multi-resistente en pediatría: Estudio de casos y controles. Revista chilena de infectología, 32(1), 19-24. Retrieved from: https://scielo.conicyt.cl/scielo.php?pid=S071 6$10182015000200003 \&$ script $=$ sci_arttext $\&$ tln $\mathrm{g}=\mathrm{p}$ 
Sánchez, M. V., Jiménez, M. A., \& de Mezerville, M. H. (2014). Acciones educativas dirigidas al personal médico y de enfermería para disminuir las infecciones del tracto sanguíneo relacionadas a catéteres venosos centrales. Enfermería Actual en Costa Rica(27). Retrieved from: https://www.redalyc.org/pdf/448/448321620 05.pdf

Torres Hernández, A., García Gómez, A., Pradere Pensado, J. C., Rives, Y. A., \& Fernández Castillo, E. (2019). Bacteriemias en la unidad de cuidados intensivos. Revista Cubana de Medicina Militar, 48(1).

Valderrama, S. L., González, P. F., Caro, M. A., Ardila, N., Ariza, B., Gil, F., \& Álvarez, C. (2016). Factores de riesgo para bacteriemia por Pseudomonas aeruginosa resistente a carbapenémicos adquirida en un hospital colombiano. Biomedica, 36(1), 69-77. Retrieved from: https://www.redalyc.org/pdf/843/843465730 10.pdf

Willis-Knishton health system. Bacteriemia en niños. (2020). Retrieved 17 Enero 2020, from: https://www.wkhs.com/healthresources/healthlibrary/article?chunkid=928946\&lang=Spani $\mathrm{sh} \& \mathrm{db}=\mathrm{hls}$

Yaguache Hernández, A. L. (2015). Procalcitonina como predictor de bacteriemia en lactantes febriles, con uroanálisis patológico, ingresados en el Hospital de Niños" Dr. Roberto Gilbert Elizalde", enero a diciembre $2014 . \quad$ Retrieved from: http://192.188.52.94/bitstream/3317/4135/1/ T-UCSG-POS-EGM-PE-30.pdf. 